\title{
Continuous refinement of the microstructure during crystallization of supercooled $\mathrm{Zr}_{41} \mathrm{Ti}_{14} \mathrm{Cu}_{12} \mathrm{Ni}_{10} \mathrm{Be}_{23}$ melts
}

\author{
J. Schroers, ${ }^{\text {a) }}$ R. Busch, ${ }^{\text {b) }}$ A. Masuhr, and W. L. Johnson \\ Keck Laboratory of Engineering Materials, California Institute of Technology, Pasadena, California 91125
}

(Received 26 January 1999; accepted for publication 8 March 1999)

\begin{abstract}
Isothermal crystallization studies are performed on $\mathrm{Zr}_{41} \mathrm{Ti}_{14} \mathrm{Cu}_{12} \mathrm{Ni}_{10} \mathrm{Be}_{23}$ melts between the liquidus and the glass transition temperature. The resulting time-temperature-transformation diagram is in good agreement with the data obtained from containerlessly processed samples. The various solidification products are examined by electron microprobe. The investigations reveal the morphology and typical length scale of the microstructure as well as the primarily solidified phases after crystallizing at different degrees of undercooling. The typical length scale decreases continuously with increasing supercooling over five orders of magnitude. The number density of nuclei during primary crystallization is estimated from the microstructure. The results are discussed within several models, such as steady-state nucleation, spinodal decomposition, and a nonpolymorphic nucleation model. (C) 1999 American Institute of Physics.
\end{abstract}

[S0003-6951(99)05118-9]

Recently discovered multicomponent metallic alloys ${ }^{1,2}$ exhibit excellent glass-forming ability. The critical cooling rate for glass formation of the $\mathrm{Zr}_{41} \mathrm{Ti}_{14} \mathrm{Cu}_{12} \mathrm{Ni}_{10} \mathrm{Be}_{23}$ (Vit 1) alloy, for example, is $1 \mathrm{~K} / \mathrm{s}^{3}$ The high thermal stability of melts of this bulk metallic glass against crystallization leads to an experimentally accessible time and temperature window to investigate isothermally the transformation behavior from the supercooled (undercooled) liquid into the crystalline phases. It was shown that the Vit 1 alloy is very robust with respect of heterogeneous surface nucleation. ${ }^{4}$ Therefore, processing of these samples in graphite crucibles is possible.

Several investigations have been performed that correlate the microstructure of metallic alloys with the nucleation and growth behavior (see, e.g., Refs. 5 and 6). Since the composition of Vit 1 is close to a deep eutectic, nucleation of crystalline phases from the homogeneous multicomponent melt is suppressed to a great extent. Therefore, it is somewhat surprising that Vit 1 crystallize at low temperatures, in the vicinity of the glass transition temperature $T_{g}$, from the amorphous state, into a nanocrystalline microstructure. Explanations, such as extremely high heterogeneous nucleation rates and quenched-in nuclei ${ }^{7}$ were applied to describe the high crystal density. Alternative processes such as phase separation in the supercooled liquid prior to crystallization ${ }^{8,9}$ or a homogeneous nucleation model, which take into account linked fluxes of interface attachment and diffusion in the liquid to the cluster neighborhood, ${ }^{10}$ were used to explain the high number density of nuclei.

This letter focuses on a detailed microstructure analysis of Vit 1 samples solidified after different isothermal annealing times at distinct levels of undercooling.

Amorphous samples were prepared by arc melting the constituents (purity ranging from $99.5 \%$ to $99.995 \%$ ) in a titanium-gettered argon atmosphere. The samples were introduced into the graphite crucibles that were inductively

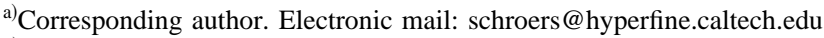

b) Permanent address: Department of Mechanical Engineering, Oregon State University, Corvallis, OR 97331. heated in a titanium-gettered argon atmosphere. The maximum cooling rates are $40 \mathrm{~K} / \mathrm{s}$. Details of the experimental setup can be found elsewhere. ${ }^{11}$ The microstructures of the solidified samples are analyzed with a Camscan Series II scanning electron microscope (SEM) as well as a Jeol JXA733 electron microprobe. The latter apparatus is equipped with five wavelength-dispersive spectrometers (WDS) that are used for determination of the various phases by element concentrations.

To get reproducible undercooling results on Vit 1, each liquid sample was overheated up to $1175 \mathrm{~K}$ prior to the isothermal undercooling experiment. Subsequently, the melt was kept at a constant undercooling level, $\Delta T=T_{\text {liq }}-T_{\text {iso }}$, with respect to the liquidus temperature. The liquidus temperature of Vit 1 is $T_{\text {liq }}=1026 \mathrm{~K}$ (Ref. 4). $T_{\text {iso }}$ denotes the isothermal temperature. The crystallization of the melt is detected from the temperature-time profile by a temperature rise due to the release of the heat of fusion during crystallization. The sample is cooled to room temperature with a rate of about $25 \mathrm{~K} / \mathrm{s}$ after the annealing procedure to conserve the solidification product.

The results of the isothermal crystallization experiments are summarized in a time-temperature-transformation (TTT) diagram shown in Fig. 1. In this diagram, the time at which a heat release is detected during isothermal annealing is plotted as a function of temperature. Additionally, data obtained by containerless electrostatic levitation (ESL) experiments ${ }^{12}$ are shown in Fig. 1 as open circles. The results obtained by ESL are in good agreement with the data from samples crystallized in graphite crucibles. The graphite crucibles do not alter the crystallization behavior in comparison to containerless processing but allow for a more reliable temperature reading, in particular, at low temperatures.

A SEM backscattering image of a sample that was undercooled for $600 \mathrm{~s}$ at $926 \mathrm{~K}(\Delta T=100 \mathrm{~K})$ is shown in Fig. 2(a). The morphology consists of a multiphase eutectic microstructure. The inset in Fig. 2(a) depicts a closer view of the microstructure. WDS measurements show that the bright needle-like phase is enriched in $\mathrm{Zr}$ and depleted in Be. X-ray 


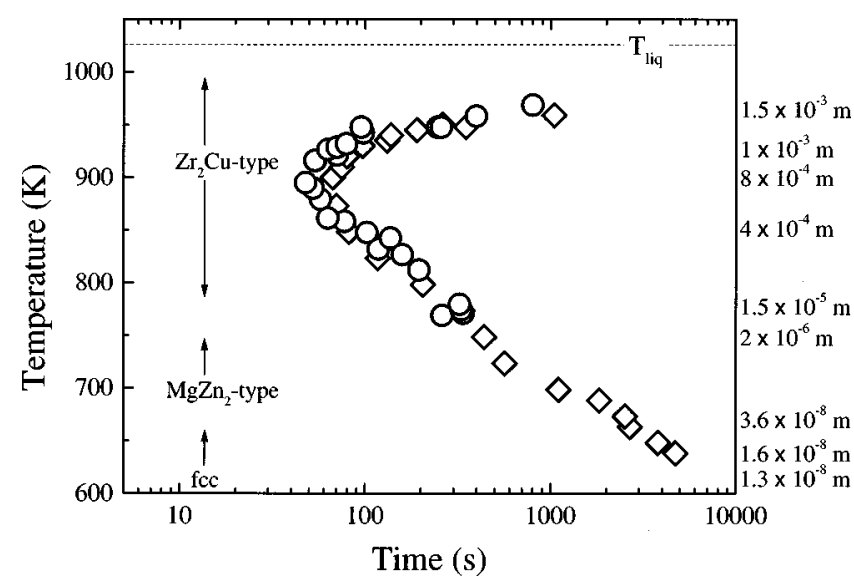

FIG. 1. Time-temperature-transformation (TTT) diagram for Vit 1. The data are measured by electrostatic levitation $(O)$ (see Ref. 12) and by processing in graphite crucibles $(\diamond)$. The temperatures of the data of Ref. 12 were corrected by a factor of 1.053 . This results from a recalibration after reassessing heating curves in the ESL (see Ref. 18). The phases solidified primarily in different temperature regions are plotted on the left-hand side of the diagram. Additionally, the length scales of the microstructures after isothermal annealing at different temperatures are shown on the right-hand side of the diagram.

studies reveal its $\mathrm{Zr}_{2} \mathrm{Cu}$-type structure. From the SEM images we conclude that the crystallization proceeds from certain nucleation events (marked by arrows). These isolated nucleation events grow until they interfere. The typical size
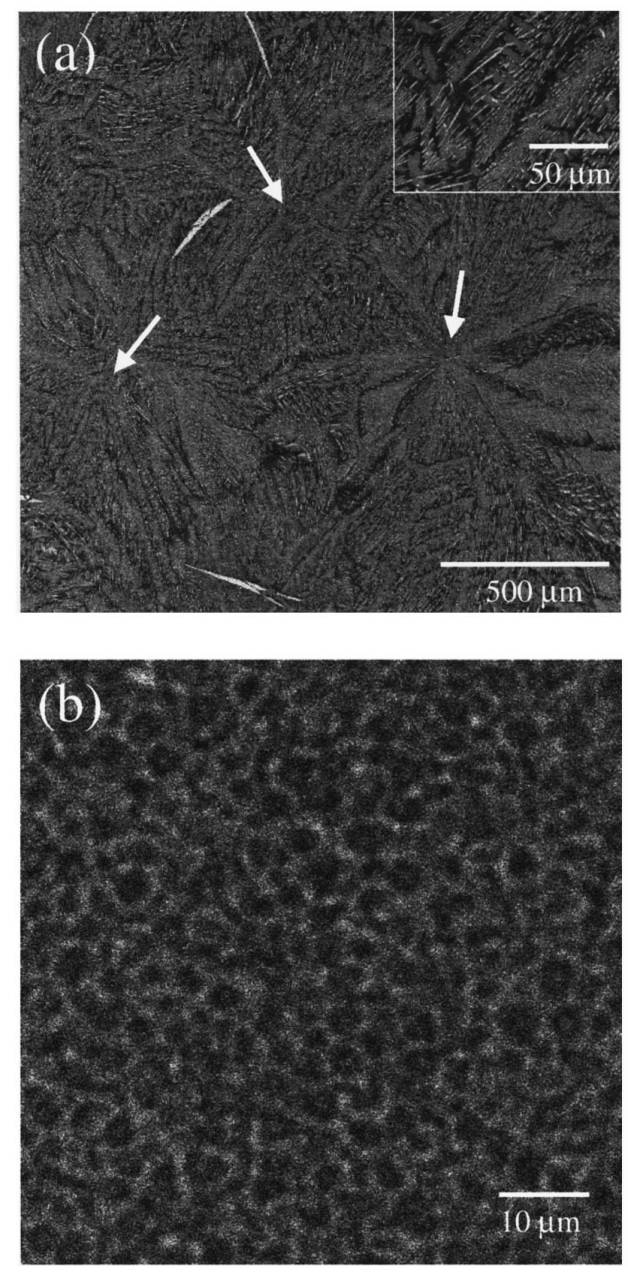

FIG. 2. SEM backscattering electron images from Vit 1 samples processed under different conditions: (a) $600 \mathrm{~s}$ at $926 \mathrm{~K}$ and (b) $200 \mathrm{~s}$ at $740 \mathrm{~K}$.

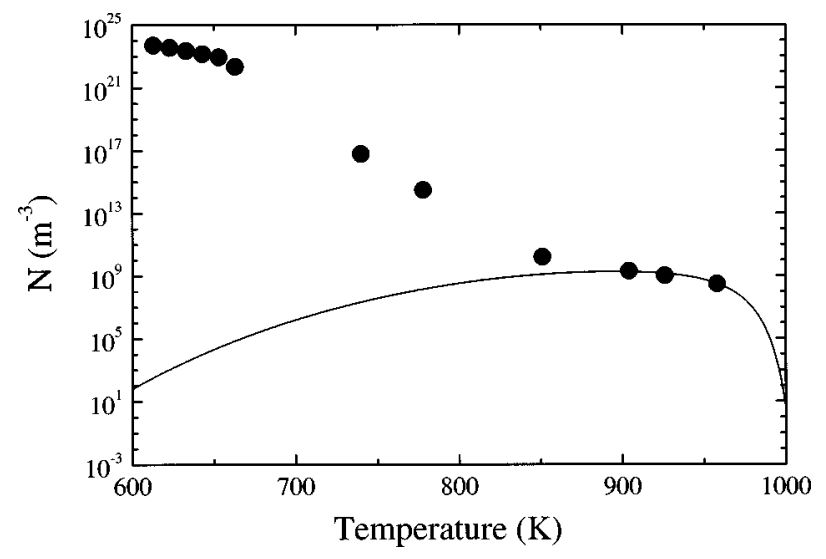

FIG. 3. Number density of nuclei $N$ determined from the microstructure of the differently processed samples ( ). The data below $700 \mathrm{~K}$ are derived from small-angle neutron scattering measurements (see Ref. 13).

of these nucleation and growth events is about $1000 \mu \mathrm{m}$ at $926 \mathrm{~K}$.

The backscattering image of a Vit 1 sample which is kept for $200 \mathrm{~s}$ at $740 \mathrm{~K}(\Delta T=286 \mathrm{~K})$ is shown in Fig. 2(b). WDS and $\mathrm{X}$-ray investigations suggest that the regions that appear dark in Fig. 2(b) consist of a phase, which is enriched in $\mathrm{Be}$ and depleted in $\mathrm{Zr}$ and has a $\mathrm{MgZn}_{2}$-type structure. The typical length scale of this crystalline microstructure is 2 $\mu \mathrm{m}$.

The length scales of the nucleation and growth events develop during different isothermal heat treatments are shown on the right-hand side of Fig. 1. In the temperature range between 958 and $740 \mathrm{~K}$ the typical length scale decreases from 1500 to $2 \mu \mathrm{m}$. The length scales below the 700 $\mathrm{K}$ annealing temperature are taken from Schneider et al. ${ }^{13}$ where the crystallization behavior of Vit 1 was studied by small-angle neutron scattering (SANS). In the temperature region from 958 to $613 \mathrm{~K}$ the length scale decreases continuously with increasing supercooling over five orders of magnitude. Figure 1 additionally shows the primarily solidified phase. In the high-temperature range, from the liquidus temperature to approximately $780 \mathrm{~K}$, a $\mathrm{Zr}_{2} \mathrm{Cu}$-type phase forms primarily, whereas between 740 and $710 \mathrm{~K}$ a $\mathrm{MgZn}_{2}$-type phase solidifies first. In the low-temperature range from 670 to $610 \mathrm{~K}$ a fcc solid-solution forms primarily. ${ }^{9}$

The number density of nuclei $N$ calculated from the size of the nucleation and growth events is plotted in Fig. 3. N increases continuously from $10^{8} \mathrm{~m}^{-3}$ at $958 \mathrm{~K}$ up to $10^{23} \mathrm{~m}^{-3}$ at $613 \mathrm{~K}$. For comparison, the solid curve represents the number density of nuclei $N=I_{\mathrm{SS}} \times t$ ( $t$ :time) taken from Ref. 14, which was applied to describe the TTT diagram. The steady-state (SS) nucleation rate $I_{\mathrm{SS}}$ is given by

$$
I_{\mathrm{SS}}=\frac{k T N_{0}}{3 \eta(T) a^{3}} \exp \left(-\frac{\Delta G^{*}}{k T}\right),
$$

in which $\Delta G^{*}$ is the activation energy to form a critical nucleus, $\eta$ denotes the viscosity, $N_{0}$ the number of potential nucleation sites, $a$ an interatomic spacing, $T$ the absolute temperature, and $k$ is Boltzmann's constant. This solid curve in Fig. 3 is in good agreement with the experimental data for $N$ at high temperatures down to $850 \mathrm{~K}$. Below $850 \mathrm{~K}$ the
to AIP license or copyright, see http://apl.aip.org/apl/copyright.jsp 
calculated and the experimental data show a very different behavior. According to steady-state nucleation [Eq. (1)], $N$ decreases continuously with decreasing temperature while the experimental data for $N$ strongly increase. At $613 \mathrm{~K}$ the experimental value is 21 orders of magnitude larger than the calculated one.

Simple transient effects are considered by

$$
I_{t}=I_{\mathrm{SS}}\left[1-\exp \left(-\frac{t}{\tau}\right)\right]
$$

with the incubation time $\tau$, given, e.g., by the expression of Davies, ${ }^{15}$

$$
\tau=6 \pi a^{3} \eta / k T .
$$

This is the time required to establish an equilibrium cluster distribution. Using viscosity data taken from Ref. 14, one can calculate $\tau$ according to Eq. (3). In the whole temperature region of interest $\tau$ is at least one order of magnitude smaller than the crystallization time. This means that the crystallization time is always much larger than the equilibration of the cluster distribution in the melt. Thus, simple transient effects according Eq. (2) can be neglected on the time scale of our crystallization process.

Obviously, simple steady-state (or transient) nucleation fails to explain the experimental finding of a continuous refinement of the microstructure with decreasing temperature. Since the microstructure investigations reveal that for different temperatures different phases solidify primarily, an attempt was made to fit the experimental data with steady-state nucleation considering three different phases for primary crystallization (see Fig. 1). The only phase-specific parameter in the steady-state nucleation rate [Eq. (1)] is the activation energy. However, even large variations of the activation energy down to some $\mathrm{eV}$ cannot describe the experimental data.

The sluggish crystallization suggested from the TTT diagram and the high nuclei density are unlikely to result from one nucleation mechanism in the homogeneous liquid. High nuclei density implies a high nucleation rate, which is in contradiction to the sluggish crystallization kinetics.

A process that is likely to describe the continuous refinement of the microstructure with decreasing isothermal temperature and the sluggish crystallization kinetics is that of a phase separation in the liquid prior to crystallization. In this case, the homogeneous liquid decomposed prior to nucleation into regions with different composition. The nucleation probability in the decomposed region is strongly increased, since composition is locally closer to the composition of the primary solidified phase. In Cahn's theory on spinodal decomposition, ${ }^{16}$ e.g., the length scale of the phase-separated region, and thus the spacing of the crystals, is mainly given by the ratio of the gradient energy and the curvature of the free energy. In the past, identification of phase separations in liquid Vit 1 prior to crystallization have been observed. ${ }^{8,9,17}$ Whether the Cahn-Hillard theory ${ }^{16}$ for spinodal decomposition is appropriate to describe the phase separation is yet unclear.

An alternative model that is based on a compositional fluctuation in the supercooled liquid is given by Kelton. ${ }^{10}$ This model for a nonpolymorphic transition takes into acDownloaded 12 Jan 2006 to 131.215.240.9. Redistribution subject count the linked stochastic fluxes of atom attachment to the cluster interface and long-range diffusion in the liquid to the cluster neighborhood. If the effective diffusion rate of a solute atom is comparable to the interfacial rate, this should cause the solute concentration in the neighborhood of small clusters to rise above that of the homogeneous phase, and therefore, result in a decomposition of the homogeneous liquid. However, it is not clear if the resulting compositional fluctuations according to this model have a well defined spacing as it was found in experiments. ${ }^{9,17}$ To determine which model is more correct, detailed studies on the timedependent behavior of the compositional fluctuations within the models and their length scales has to be performed.

In conclusion, isothermal crystallization experiments of Vit 1 alloys were performed in a supercooled liquid. The primarily solidified phase as well as the typical length scales for nucleation and growth events in samples processed at distinct levels of undercooling were determined from the microstructure. The length scale decreases continuously by five orders of magnitude upon lowering the annealing temperature from 958 to $613 \mathrm{~K}$. The number of nucleation events at a given undercooling was determined from the microstructure and was found to increase from $10^{8} \mathrm{~m}^{-3}$ for samples processed at $958 \mathrm{~K}$ to $10^{23} \mathrm{~m}^{-3}$ for samples processed at 613 $\mathrm{K}$. This indicates a high nucleation rate and appears to be in conflict with sluggish kinetics suggested by the long times in the TTT diagram. This apparent contradiction can be qualitatively explained by decomposition in the liquid state prior to crystallization.

This work was supported by the National Aeronautics and Space Administration (Grant No. NCC8-119) and the Department of Energy (Grant No. DEFG-03086ER45242).

${ }^{1}$ A. Inoue, T. Zhang, and T. Masumoto, Mater. Trans., JIM 31, 177 (1990).

${ }^{2}$ A. Peker and W. L. Johnson, Appl. Phys. Lett. 63, 2342 (1993).

${ }^{3}$ Y. J. Kim, R. Busch, W. L. Johnson, A. J. Rulison, and W. K. Rhim, Appl. Phys. Lett. 65, 2136 (1994).

${ }^{4}$ A. Masuhr, R. Busch, and W. L. Johnson, Mater. Sci. Forum 269-272, 779 (1998).

${ }^{5}$ U. Köster and J. Meinhardt, Mater. Sci. Eng., A 178, 271 (1994).

${ }^{6}$ H. J. Fecht, Mater. Res. Soc. Symp. Proc. 455, 307 (1997).

${ }^{7}$ J. C. Foley, D. R. Allen, and J. H. Perepezko, Scr. Mater. 35, 655 (1996).

${ }^{8}$ R. Busch, S. Schneider, A. Peker, and W. L. Johnson, Appl. Phys. Lett. 67, 1544 (1995).

${ }^{9}$ S. Schneider, P. Thiyagarajan, and W. L. Johnson, Appl. Phys. Lett. 68, 493 (1996).

${ }^{10}$ K. F. Kelton, Philos. Mag. Lett. 77, 337 (1998).

${ }^{11}$ A. Masuhr, Ph.D. thesis, California Institute of Technology (1998).

${ }^{12}$ Y. J. Kim, R. Busch, W. L. Johnson, A. J. Rulison, and W. K. Rhim, Appl. Phys. Lett. 68, 1057 (1996)

${ }^{13}$ S. Schneider, P. Thiyagarajan, U. Geyer, and W. L. Johnson, Mater. Res. Soc. Symp. Proc. 455, 295 (1997).

${ }^{14}$ A. Masuhr, T. A. Waniuk, R. Busch, and W. L. Johnson, Phys. Rev. Lett. 82, 2290 (1999)

${ }^{15}$ H. A. Davies, in Proceedings of the Third International Conference on Rapidly Quenched Metals, edited by B. Cantor (Chameleon, Brighton, 1978), pp. 1-21.

${ }^{16}$ E. L. Huston, J. W. Cahn, and J. E. Hilliard, Acta Metall. 14, 1053 (1966).

${ }^{17}$ W-H. Wang, Q. Wei, S. Friedrich, M. P. Macht, N. Wanderka, and H. Wollenberger, Appl. Phys. Lett. 71, 1053 (1997).

${ }^{18}$ Y. J. Kim, R. Busch, W. L. Johnson, A. J. Rulison, and W. K. Rhim, in the 7th International Symposium on Experimental Methods for Microgravity Material Science, TMS Meeting, Las Vegas, February 13-15, edited by R. A. Schiffman (1995), p. 153. 